\title{
A Brief Analysis on the Characteristics of Chinese Medicine and Treatment for Cough Variant
}

\author{
Yuntao Duan', Junming Hou' ${ }^{*}$, Yang Hui', Dezhen Yang² \\ 1Shaanxi University of Chinese Medicine, Xi Xian New Area 712046, Shaanxi Province, China \\ ${ }^{2}$ Affiliated Hospital of Shaanxi University of Traditional Chinese Medicine, Xianyang 712000, Shaanxi Province, China \\ *Corresponding author: Junming Hou, houjunming078@126.com
}

\begin{abstract}
Cough variant is a common disease of the respiratory system and can lead to a unique type of asthma. The disease has no obvious symptoms such as wheezing nor shortness of breath. Coughing is the main clinical symptom, as it causes airway hyper-responsiveness. Traditional Chinese medicine (TCM) has a unique understanding of this disease, and the effect is obvious after treatment, as it's based on symptom differentiation. This article takes the concept of "mild fluid retention" from "The Synopsis of the Golden Chamber" as an entry point, briefly describes the relationship between cough variant and mild fluid retention, as well as the diagnosis and treatment of Chinese medicine.
\end{abstract}

Keywords: Cough variant asthma; Cough; Mild fluid retention; Resolving fluid retention with warm medicine

Publication date: July 2021; Online publication: July 31, 2021

\section{Introduction}

\subsection{The concept of mild fluid retention}

Mild fluid retention was first seen in "The Synopsis of the Golden Chamber: Phlegm-fluid retention and cough abnormal pulse identification and treatment." Additionally, "One's mild fluid retention due to coughing should be discharged by urinating, Linggui Shugan decoction is the main treatment for that." It belongs to a kind of fluid retention. Combining with the discussion in the same article, "When one eats less and stores a lot of fluid, fluid retentions in epigastrium, causing sufferings different from palpitation to shortness of breath," it can be known that mild fluid retention is a mild symptom of fluid retention, and these are typical symptoms of shortness of breath.

Mild fluid retention is one of fluid retention pathogen applicable to phlegm-fluid retention treatment principle-sick patients with phlegm-fluid retention should be treated with warm medicine." which more clearly points out the nature of fluid retention, among which the word "phlegm" is changed from the word "light" by later generations. The original meaning is "the appearance of cold water shaking" [1]. The phlegm-fluid retention (light retention) described by Zhang Zhongjing is an abnormal fluid retention caused by the unfavorable metabolism of human water and fluids. As summarized by Zhao Ding et al. "2], "phlegmfluid retention" is usually a result of yang deficiency with yin exuberance as well as asthenia in origin and asthenia in superficiality. The formation is attributed to the lack of Yang in the lungs, spleen, and kidneys. Mild fluid retention in phlegm-fluid retention and cough abnormal pulse identification and treatment in the Synopsis of the Golden Chamber belongs to this type of fluid retention pathogen, and is consistent in nature and pathogenesis with phlegm-fluid retention. As the external symptom of mild fluid retention, shortness of breath is caused by mild fluid retention hindering the up and down of the Qi mechanism. Mild fluid retention blocks the rise and fall, hinders the gasification, causing the movement of the air machine and the water fluid to be out of balance, fluid retentions in the chest and diaphragm, as it affects the lungs, and can 
also cause lung failure and lung Qi imbalance. Therefore, in addition to shortness of breath, coughing is also one of the common clinical symptoms.

In the "Golden Chamber Synopsis," the characteristics of mild fluid retention can be summarized as follows ${ }^{[3]}$ :

(1) Conceptually, mild fluid retention refers to the slight fluid retention.

(2) The reason is nothing more than the inability of Yang Qi, which is used according to the provisions. Prescriptions may be insufficient for spleen and kidney Yang Qi.

(3) The pathological change is the internal resistance of fluid retention, resulting in abnormal Qi movement.

(4) The typical clinical manifestation is shortness of breath, cough, dizziness, and difficulty in urination.

(5) Treatment Zhang Zhongjing argued that "it should be removed by urinating," and its connotation is to transform gas and water, so that pathogen has a way to go.

\section{The main cause of cough variant is mild fluid retention}

The main symptom of cough variant is cough, which can be classified into the category of "cough" in Chinese medicine, but it is more unique than normal cough. The main cause of cough variants diagnosed by western medicine and TCM, is mild fluid retention. Western medicine and TCM are consistent in symptoms and disease evolution.

In terms of symptoms, cough is a common symptom of mild fluid retention, while cough variant is mainly cough with shortness of breath, which is very consistent with the characteristics of mild fluid retention. Some patients often have symptoms such as sneezing and running nose when they get sick. It has the characteristics of a cold, which is consistent with the characteristics of mild fluid retention described in the previously, while cough variant has a longer course and can be induced by wind-cold and irritating factors. Symptoms such as cough are often worse at night. Fluid retention, belonging to Yin pathogen, hiding in the internal, is easy to be induced by the external pathogen, and also worsens during the Yin time.

In the evolution of the disease, some patients first have allergic symptoms such as allergic rhinitis (allergic rhinitis), which develops the coughs. When poorly controlled, it can further develop into asthma. Combining cough variant and asthma in symptomatic manifestations, the similarities and differences in the attack pattern show that cough variant is closely related to asthma and has a progressive degree. Wang Lie, a master of TCM, suggested that the name of the medical disease was "asthma" because of the rule of "longterm cough and stagnation of phlegm and dysfunction" [4].

In the understanding of modern medicine, the relationship between cough variant and typical asthma has also been clarified. The latter is considered to be the progressive stage of cough variant ${ }^{[5]}$, and both have airway inflammation in the pathogenesis. As a pathological body fluid, inflammatory exudate can also be classified as phlegm in TCM, which can be considered as a substantial symptom of mild fluid retention.

\section{Resolving the fluid retention with warm medicine drinking is the key to the treatment of cough variant}

The treatment of mild fluid retention has the argument of "it should be removed by urinating," which gives the idea of letting out the phlegm by drinking warm medicine, to allow a normal path of water and liquid transport ${ }^{[6]}$, the application of Linggui Shugan decoction and Shenqi pills can help with this treatment. Regarded by later generations as a classic case of treating the same disease with different treatments, its essence is the method of examining the evidence and seeking the cause and guiding the pathogens according to the situation. It is also in line with the treatment principle of "sick patients with phlegm-fluid retention should be treated with warm medicine."

Therefore, in the treatment of mild fluid retention, warming medicine and decoction is the key. Zhang 
Zhongjing's warming medicine can be used, such as Xiaoqinglong decoction, Houpu Mahuang decoction and Zhenwu decoction for cough. Zhang Zhongjing uses dried ginger, the combination of Asarum and Schisandra is used to relieve cough. In Shegan Mahuang decoction, Asarum, Schisandra and ginger are used, the addition and subtraction guidance of Xiaochaihu Tang and Sini San also shows that people who cough should use dried ginger and Schisandra. It can be seen that dried ginger (ginger), Asarum, Schisandra and other medicines not only conform to the treatment principle of warm herbal medicine and western formulated medicine, but also correspond to the symptoms of cough and shortness of breath, which are the key to the treatment of cough variants.

\section{Summary}

In summary, mild fluid retention is a mild fluid retention disease, which is closely related to cough variants. Mild fluid retention is the main cause of cough variants, and it is the "ratoon" of recurrent cough in this disease. Resolving the fluid retention with warm medicine is the key to treating cough variant.

\section{Disclosure statement}

The author declares no conflict of interest.

\section{References}

[1] Dai ZY, 2010, The Concept of "Phlegm" and the Differential Diagnosis of Phlegm-Fluid Retention and Water-Dampness: Research Inspiration from Japan. Proceedings of the Symposium on Diagnostics of Chinese Medicine in Japan and China, 6.

[2] Zhao D, Lv CX, 2017, Discussion on "Patients with Phlegm-fluid Retention Should be Treated with Warm Medicine.” Chinese Journal of Traditional Chinese Medicine, 2017, 32(5): 2229-2232.

[3] Zhang LS, Zhou SZ, Li C, 2017, Mild fluid retention and Chronic Cough. Chinese Journal of Traditional Chinese Medicine, 32(7): 2998-3000.

[4] Li XY, Zhang H, 2015, Professor Wang Lie's Academic Experience in Treating Children with Asthma. Journal of Pediatrics of Chinese Medicine, 11(4): 1-3.

[5] Gao LX, Yan Y, Bao HP, et al., 2019, Modern Research Progress on Cough Variant Asthma. Chinese Journal of Traditional Chinese Medicine, 34(9): 4171-4174.

[6] Wu ZX, Lian JW, Jiang YN, 2014, A Detailed Analysis of the Meaning of the Article: “One's Mild Fluid Retention due to Coughing Should be Discharged by Urinating." in "The Synopsis of the Golden Chamber." Journal of Zhejiang University of Traditional Chinese Medicine, 38(4):376378. 